\title{
BMJ open Asperger syndrome and anxiety disorders (PAsSA) treatment trial: a study protocol of a pilot, multicentre, single-blind, randomised crossover trial of group cognitive behavioural therapy
}

\author{
Peter E Langdon, ${ }^{1}$ Glynis H Murphy, ${ }^{2}$ Edward Wilson, ${ }^{3}$ Lee Shepstone,${ }^{3}$ \\ David Fowler, ${ }^{4}$ David Heavens, ${ }^{4}$ Aida Malovic, ${ }^{2}$ Alexandra Russell ${ }^{1}$
}

To cite: Langdon PE, Murphy GH, Wilson E, et al. Asperger syndrome and anxiety disorders (PAsSA) treatment trial: a study protocol of a pilot, multicentre, single-blind, randomised crossover trial of group cognitive behavioural therapy. BMJ Open 2013;3: e003449. doi:10.1136/ bmjopen-2013-003449

- Prepublication history for this paper is available online. To view these files please visit the journal online (http://dx.doi.org/10.1136/ bmjopen-2013-003449).

Received 20 June 2013 Accepted 27 June 2013

\footnotetext{
${ }^{1}$ Department of Psychological Sciences, Norwich Medical School, University of East Anglia and Broadland Clinic, Hertfordshire Partnership NHS Foundation Trust, Norwich, UK

${ }^{2}$ Tizard Centre, University of Kent, Canterbury, UK ${ }^{3}$ Department of Population Health and Primary Care, Norwich Medical School, University of East Anglia, Norwich, UK

${ }^{4}$ Norfolk and Suffolk NHS Foundation Trust, Norwich, UK
}

Correspondence to Dr Peter E Langdon; P.Langdon@uea.ac.uk

\section{ABSTRACT}

Introduction: A number of studies have established that children, adolescents and adults with Asperger syndrome (AS) and high functioning autism (HFA) have significant problems with anxiety. Cognitive behavioural therapy (CBT) is an effective treatment for anxiety in a variety of clinical populations. There is a growing interest in exploring the effectiveness of CBT for people with AS who have mental health problems, but currently there are no known clinical trials involving adults with AS or HFA. Studies with children who have AS have reported some success. The current study aims to examine whether modified group CBT for clinically significant anxiety in an AS population is likely to be efficacious.

Methods and analysis: This study is a randomised, single-blind crossover trial. At least 36 individuals will be recruited and randomised into a treatment arm or a waiting-list control arm. During treatment, individuals will receive 3 sessions of individual CBT, followed by 21 sessions of group CBT. Primary outcome measures focus on anxiety. Secondary outcome measures focus on everyday social and psychiatric functioning, additional measures of anxiety and fear, depression, health-related quality of life and treatment cost. Assessments will be administered at pregroup and postgroup and at follow-up by researchers who are blinded to group allocation. The trial aims to find out whether or not psychological treatments for anxiety can be adapted and used to successfully treat the anxiety experienced by people with AS. Furthermore, we aim to determine whether this intervention represents good value for money. Ethics and dissemination: The trial received a favourable ethical opinion from a National Health Service (NHS) Research Ethics Committee. All participants provided written informed consent. Findings will be shared with all trial participants, and the general public, as well as the scientific community.

Trial Registration: ISRCTN 30265294 (DOI: 10.1186/ISRCTN30265294), UKCRN 8370.

\section{ARTICLE SUMMARY}

Article focus

- People with autistic spectrum disorders have higher rates of mental health problems than the general population.

- The aims of this trial are (1) to develop groupbased cognitive behavioural therapy (CBT) for people with Asperger syndrome and high functioning autism who have anxiety disorders, (2) to asssess the efficacy of the intervention through a pilot, single-blind, crossover randomised control trial and (3) to estimate the costeffectiveness of the CBT intervention compared to no intervention.

Key messages

- This study is a pilot trial which will determine whether or not group CBT is likely to be an effective intervention for anxiety disorders among people who have autistic spectrum disorders.

- The trial will also determine whether the intervention is cost-effective.

- The study will also examine the views participants have about the intervention.

Strengths and limitations of this study

- Appropriate randomisation and blinding have been used.

- The study makes use of several different types of clinical assessment methods.

- However, the sample size is relatively small, but nevertheless should be sufficient, bearing in mind that little is known about the effectiveness of CBT for mental health problems among adults with autistic spectrum disorders.

\section{BACKGROUND}

Anxiety-related symptomology is commonly found in those with autistic spectrum disorders (ASDs). ${ }^{1-4}$ Prevalence studies indicate 
that around $13.6 \%$ of children with Asperger syndrome (AS) suffer from clinically relevant anxiety ${ }^{5}$ and up to $45 \%$ of adolescents with AS have significant difficulties with generalised anxiety, social anxiety or specific phobias. ${ }^{6}$ A recent study found that in a sample of young adults with AS, around $50 \%$ had an anxiety disorder, ${ }^{7}$ while other evidence suggests that people with ASDs are quite likely to develop difficulties across a range of anxiety disorders (eg, panic disorder and generalised anxiety disorder). ${ }^{8} 9$

Recent guidance creates standards for the diagnosis and management of adults with ASDs, ${ }^{10}$ and while there are an increasing number of adults being diagnosed with ASDs, the National Health Service (NHS) in the UK is charged with meeting the mental health needs of this population. Although currently there are no known data relating to the cost of AS and anxiety for the UK, the lifetime cost of ASDs is thought to be in the region of $£ 2.4 \mathrm{million} /$ person, with the total cost to the UK society exceeding $£ 1$ billion/year. ${ }^{11}$ The weekly societal cost of providing care to a person with an ASD in 19992000 was estimated to be in the region of $£ 855.00$, with an associated parental loss of income estimated to be in the region of $£ 275.00 /$ week. $^{12}$ In view of this, effective treatments for anxiety in this population may lead to significant improvements in psychosocial functioning, independence and quality of life.

Cognitive behavioural therapy (CBT) has been shown to be an effective treatment for anxiety in people without ASDs. ${ }^{13}{ }^{14}$ As a consequence, there is a growing interest in examining the utility of CBT for people with ASDs who experience difficulties with anxiety. Adapted CBT has been shown to be a successful treatment for anxiety disorders among children with ASD. ${ }^{15-19}$ Cardaciotto and Herbert ${ }^{20}$ and Weiss and Lunsky ${ }^{21}$ undertook some case study work suggesting that CBT may be helpful for adults with AS. In a recent randomised controlled trial (RCT), mindfulness-based therapy was found to reduce anxiety symptoms in adults with ASD, although it is unclear whether or not the assessors were blind within this study. ${ }^{22}$ At present, there appears to be no published randomised, single-blind control trials investigating the effectiveness of group CBT for adults with ASD and anxiety disorders.

The government autism strategy, 'fulfilling and rewarding lives', recognises that children and adults with ASD are excluded from many NHS services, including mental healthcare. ${ }^{23}$ The current study aims to help achieve the goals set within this strategy by contributing to the creation of a knowledge base. This will help inform clinical practice and service delivery for the treatment of adults with AS who experience problems with anxiety. In line with the recommendations made by the National Institute for Health and Care Excellence (NICE) ${ }^{10}$ it will also examine whether CBT is cost-effective for the treatment of anxiety among adults with ASD. Roberge et $a l^{24}$ argue that group CBT is more cost-effective than individual CBT in patients with some types of anxiety disorders and a similar approach has been adopted within the current study.

The key aims are (1) To develop a modified, groupbased CBT intervention manual for adults with AS and clinical problems with anxiety; (2) to assess the efficacy of the CBT intervention through a pilot, single-blind, crossover RCT in adults with AS experiencing problems with anxiety and (3) to estimate the cost-effectiveness of the CBT intervention compared with no intervention. Assessment of efficacy will include assessments of anxiety and depression symptomology, as well as social and occupational functioning.

\section{METHOD}

\section{Design and randomisation}

This study is a single-blind, randomised crossover trial. Owing to the nature of the intervention, therapists and participants cannot be blinded to treatment allocation. However, researchers who carry out the assessments will be blinded in order to minimise bias. Participants will be allocated to the treatment arm (group CBT) or the waiting-list control arm using block randomisation with even pairs. The randomisation will be stratified by study site. The therapists at each research site will contact participants to inform them of their group allocation, while blinded researchers will complete the assessments with participants. All of the randomisation procedures are to be completed by the Norfolk and Norwich University Hospitals NHS Foundation Trust, Clinical Research and Trials Unit based at the University of East Anglia.

After 24 weeks of treatment, the two arms will cross over and those within the waiting-list control group will receive 24 weeks of treatment, while those who have already had treatment will be assessed again following this second period of treatment. This will allow for a 6-month follow-up of $50 \%$ of the participants within the trial.

\section{Allocation concealment}

Adequate allocation concealment will be ensured using an onsite computer system with allocations kept in a locked, unreadable computer file. Only the therapists will access this after enrolled participants have been entered into the computer system.

\section{Implementation}

Participants will go through a screening process conducted by researchers. This will ensure that they meet the inclusion criteria before they are enrolled into the study. Randomisation will be computer generated. The software written to enable randomisation will use a programming system called VB.NET/ASP.NET and the underlying database will be SQL Server 2005 .

\section{Blinding}

At least three researchers will be blind to the randomisation process and group allocation. They will remain 
blind during the postgroup and follow-up assessments. Participants will be regularly reminded not to disclose their group allocation to these researchers throughout their involvement in the study. All participants and the therapists will be aware of the group allocation.

\section{Participants}

Thirty-six individuals will be recruited throughout Kent, South East London and Norfolk within the UK. Recruitment will be through learning disability teams, Asperger/autism teams, AS user groups, such as Asperger East Anglia, the Kent Autistic Trust, Bridging the Gap, the Disability and Dyslexia Support Services at the University of Kent, and adult mental health teams.

The inclusion criteria are: (1) participants fulfil diagnostic criteria for AS, high functioning autism or pervasive developmental disorder not specified; (2) participants have clinically significant difficulties with anxiety as confirmed through the use of the Hamilton Rating Scale for Anxiety, with a score of $>14$ qualifying for inclusion; (3) participants are between 16 and 65 years of age and (4) Full Scale Intelligence Quotient $>70$. The exclusion criteria are (1) participants suffering from post-traumatic stress disorder, or anxiety related to substance misuse; (2) comorbid severe psychiatric disorders that impair capacity to consent to take part (eg, psychosis); and (3) current substance abuse, such as alcohol or drugs.

\section{Sample size}

Bearing in mind that this is a pilot study, and considering that this study will not be a definitive RCT concerning the clinical effectiveness of this intervention, a sample size of 36 has been chosen. If we take the Hamilton Anxiety Rating Scale as our primary outcome measure, and consider that other trials of CBT for anxiety disorder have reported between a $66 \%$ and $72 \%$ change on this measure as a consequence of psychological treatment, ${ }^{25-27}$ then our study is likely to have a large effect size. However, we do not know whether the clinical effectiveness of group CBT is similar for people with AS. Once this study is completed, we will be able to estimate the sample size needed for a definitive trial.

\section{Ethical considerations}

Informed consent will be sought from participants and their carers. Research workers are responsible for providing study information to participants and obtaining consent; all participants are afforded time to consider whether they wish to participate and are given the opportunity to ask any questions. Information about the study was provided in an 'easier to read' format for participants who may have reading difficulties. Participants may withdraw from the trial at any stage without giving a reason. The withdrawal will not affect access to other treatments or services. Participants will have the contact details of the study team and are able to make contact and ask any questions. Should they wish to do so, participants who withdraw from the trial will be able to complete the scheduled outcome assessments. All information disclosed and data collected in the study will be confidential, and individuals will not be identifiable in any published material. In line with standard operating procedures, the reporting of serious adverse events will be made to the Chief Investigator, who, together with the study sponsor (Norfolk and Suffolk NHS Foundation Trust), will follow national procedures for reporting such incidents to the NHS ethics committee.

\section{Intervention}

The intervention used within this study comprises 24 weekly sessions, each lasting approximately $1 \mathrm{~h}$. Those in the treatment arm will receive three initial sessions of 1:1 CBT, followed by 21 group CBT sessions. The initial three sessions of therapy will aim to help socialise each participant into CBT and to address any concerns they may have about joining the group.

In order to ensure adherence to treatment, a treatment manual $^{28}$ was developed with specific aims for each session, and all sessions will be delivered by a registered clinical psychologist or a qualified cognitive behavioural therapist. The treatment manual includes the following topics (1) cognitive restructuring, (2) anxiety management techniques, (3) systematic desensitisation, (4) exposure to feared social situations and (4) social skills training. Cognitive restructuring refers to the process of learning to identify unhelpful cognitions, which may trigger or maintain anxiety, and then learning to challenge or replace these cognitions with more appropriate appraisals. Anxiety management techniques refer to progressive relaxation training, learning to identify anxiety-related triggers and the development of appropriate techniques to successfully manage anxiety. Systematic desensitisation and exposure to feared social situations involve the gradual exposure of the participant in a safe manner to social situations that provoke fear. These techniques allow participants to learn that anxiety is not harmful and help to reduce avoidance to feared situations. Finally, social skills training refers to the actual teaching of social skills including how to understand verbal and non-verbal social cues, and appropriate social interaction across contexts. These skills will be practiced in vivo. In addition to the intervention, participants in both arms before and after crossover were permitted to engage in treatment as usual (TAU).

\section{Outcome measures and analysis}

All outcome measures will be administered by the researchers at baseline (prior to any treatment), after week 24 (follow-up 1) and after week 52 (follow-up 2). Besides the primary and secondary measures at baseline, we will record individual demographics, which includes their age, gender, ethnic group, diagnostic history, current medication and any prior treatment. 


\section{Primary outcome measures}

Social phobia inventory

This is a 17-item self-report measure of behavioural, physiological and cognitive symptoms associated with social phobia. ${ }^{29}$ Participants will rate the frequency with which they experience each symptom over the past week, using a five-point Likert-type scale (0-4).

\section{Liebowitz social anxiety scale}

This instrument is a self-report scale that assesses fear and avoidance throughout 24 listed situations, which are likely to elicit social anxiety. ${ }^{30}$

\section{Hamilton rating scale for anxiety}

This is a structured clinician-rated scale incorporating 14 factors, which will be considered as valid indicators of anxiety. ${ }^{31}$ Each factor reflects a symptom of anxiety; physical as well as mental symptoms are represented. The factors are scored on a five-point scale as part of a structured interview.

\section{Secondary outcome measures}

Social and emotional functioning interview (informant and subject versions)

A semistructured clinician-rated assessment of everyday social and psychiatric functioning will be conducted, which is designed to assess independence, leisure, interpersonal problems, employment and social relationships. Some items are shared with the Autistic Diagnostic Observation Schedule. $^{32}$

\section{Social Interaction Anxiety Scale}

This self-report 20-item scale is a measure of anxiety associated with social anxiety and social phobia in accordance with the Diagnostic and Statistical Manual of Mental Disorders, Fourth Edition (DSM-IV) criteria. ${ }^{33} 34$ Experiences will be rated on a five-point scale from 0 (not at all characteristic of me) to 4 (extremely characteristic of me).

\section{Fear questionnaire}

This is a self-report questionnaire about individual perception of fears and phobias in which respondents will be asked what will be the likelihood of their avoiding a list of situations, due to anxiety/fear or any other unpleasant feelings. In addition to the 15 pre-existing items, the individual will be asked to document and score any individual phobias they would wish to be treated. $^{35}$

\section{Hamilton rating scale for depression}

This structured clinician-rated interview is considered a valid indicator of depression and the ratings will be based on the interviewer's objective and subjective perceptions during the assessment. ${ }^{36}$ Eight items are scored on a 0 (not present) to 4 (severe) point scale, and nine items are scored from 0 to 2 (levels of severity).
SF-36 (http://www.sf-36.org) and EQ-5D (http://www. eurogol.org)

These two self-report instruments will be used to assess overall health-related quality of life (HRQoL). Summary scores from the SF-36 and EQ-5D questionnaires will be used to estimate health profiles as well as an index of overall HRQoL (utility) using standard algorithms relevant to the UK population. ${ }^{37} 38$

\section{Resource use and cost}

Health service use, patient's out-of-pocket costs and the value of any lost productivity to society will be collected using an adapted version of the client service receipt inventory. This is a tool specifically designed to measure patient resource use in the area of mental health, although it has subsequently been adapted to other areas too. Resource use involved in delivering the intervention (ie, conducting the group-based sessions) will be based on time records for professionals and participants. Resource use estimates will be multiplied by unit costs of services extracted from standard NHS sources (eg, unit costs of health and social care ${ }^{39}$ and National Schedule of Reference Costs ${ }^{40}$ ). Where national estimates are not available, attempts will be made to obtain representative local or regional unit costs. A common price-year will be used throughout to be determined at the point of analysis. As the follow-up period for this trial is less than 1 year, discounting of costs or outcomes is not necessary.

\section{Statistical methods}

Data will be analysed using repeated measures statistics (eg, repeated measures analysis of variance and analysis of covariance) making use of intent-to-treat analysis as appropriate. We may wish to control for various variables in this analysis, for example differences in baseline anxiety scores. Summary scores from the SF-36 and EQ-5D questionnaires will be used to estimate health profiles as well as an index of overall HRQoL (utility). SF-36 and EQ-5D results will be converted to utilities using the SF- $6 \mathrm{D}^{37}$ and multiview harmonium ${ }^{38}$ algorithms, respectively. Quality-adjusted life years (QALYs) gained will be estimated from the resulting area under the curves.

Collection of SF-36 and EQ-5D will allow a comparison of the performance of the two instruments in this pilot study to update data collection tools for future full trials. Costs will be analysed from the perspectives of the public sector (NHS and social services) and society, and based on the resource use gleaned from the resource use questionnaire multiplied by unit costs as described above.

Within-trial economic analyses will report the mean cost and outcome, incremental cost and outcome and incremental cost-effectiveness ratios (ICERs) over a time horizon of 24 weeks (ie, comparing approximately 6 months of group CBT vs 6 months of 'TAU'). Outcomes in the economic analysis will be the Social and Emotional Functioning Interview score and QALYs gained (estimated using the EQ-5D and SF-6D algorithms). Uncertainty around the point estimate of the 
ICER will be presented as a cost-effectiveness acceptability curve. ${ }^{41}$ Additional modelling analyses will extend the time horizon to 5 years based on the extrapolation of trial costs and outcomes to the longer time horizon (and making use of the 24-week follow-up data in the intervention arm). Different assumptions as to the duration of effectiveness of treatment will be compared in a series of scenario analyses.

\section{DISCUSSION}

Recently published NICE guidelines ${ }^{10}$ call for greater support and service planning for those with ASDs. Despite the high prevalence of anxiety in this population, there are few studies investigating appropriate intervention. This study aims to investigate the efficacy of CBT for anxiety in adults with ASDs.

This study will involve developing and trialling modified group CBT specifically for adults with ASDs experiencing problems with anxiety. The use of CBT may lead to a reduction in anxiety and an increase in social and occupational functioning among adults with ASDs. The study will also investigate whether the use of group CBT is a cost-effective approach in treating anxiety in this population. This is not a definitive trial, and although we aim to determine whether or not CBT is likely to be effective, once treatment is completed, participants will be invited to take part in further interviews in order to ascertain their personal views about the intervention.

\section{Trial status}

The trial is currently closed to recruitment, and all sites have crossed over. Data collection is scheduled to finish in January 2014.

Acknowledgements The authors would like to express their marked gratitude to all those taking part part in this trial, including all those on the Steering Group. The authors also extend their warm regards to Asperger East Anglia (http://www.asperger.org.uk), the Kent Autistic Trust (http://www.kentautistic. com), Bridging the Gap support group and the Disability and Dyslexia Support Services at the University of Kent (http://www.kent.ac.uk/ddss). Further, the authors would like to thank Robyn Steward (http://www.robynsteward.com) who is also a member of the Steering Group and provided expert comments on some trial documentation, and Anthony Dyer, Data Manager, Clinical Trials Unit who has been exceptional in giving support and advice.

Contributors PEL was responsible for the design and conduct of this trial. He authored this manuscript, the treatment manual and acted as a therapist. GHM also contributed to the design and conduct of this trial, authored the treatment manual and acted as a therapist. PEL and GHM jointly conceived the study. EW was responsible for the design and analysis of the health economics aspects of this trial and authored this manuscript. LS was responsible for the statistical analysis of this trial and helped construct the design. DF helped to construct the design of this trial and provided advice on the intervention. $\mathrm{DH}, \mathrm{AM}$ and $\mathrm{AR}$ are employed as researchers for the trial and drafted this manuscript. DH coauthored the intervention with PEL and GHM. All authors read and approved the final version of the manuscript.

Funding This article presents independent research funded by the National Institute for Health Research (NIHR) Research for Patient Benefit programme (Grant Reference: PB-PG-1208-18024). The views expressed are those of the author(s) and not necessarily those of the National Health Service (NHS), the National Institute for Health Research or the Department of Health. PEL is funded by a National Institute for Health Research Postdoctoral Fellowship.
Competing interests None.

Ethics approval Cambridge 4 NHS Ethics Committee.

Provenance and peer review Not commissioned; internally peer reviewed.

Data sharing statement The current unpublished data for this study are available to PEL, GHM, EW, LS and DF. However, data collection has not yet been completed.

Open Access This is an Open Access article distributed in accordance with the Creative Commons Attribution Non Commercial (CC BY-NC 3.0) license, which permits others to distribute, remix, adapt, build upon this work noncommercially, and license their derivative works on different terms, provided the original work is properly cited and the use is non-commercial. See: http:// creativecommons.org/licenses/by-nc/3.0/

\section{REFERENCES}

1. Rescorla LA. Preschool psychiatric disorders: diagnostic classification and symptom patterns. J Am Acad Child Adolesc Psychiatry 1986;25:162-9.

2. Russell E, Sofronoff K. Anxiety and social worries in children with Asperger syndrome. Aust N Z J Psychiatry 2005;39:633-8.

3. Szatmari $P$, Archer $L$, Fisman $S$, et al. Asperger syndrome and autism: differences in behavior, cognition and adaptive functioning. J Am Acad Child Adoles Psychiatry 1995;34:1662-70.

4. Tantam D. Lifelong eccentricity and social isolation. Part 2: Asperger syndrome or schizoid personality disorder. $\mathrm{Br} J$ Psychiatry 1988;153:783-91.

5. Kim JA, Szatmari P, Bryson SE, et al. The prevalence of anxiety and mood problems among children with autism and Asperger syndrome. Autism 2000;4:117-32.

6. Green J, Gilchrist A, Burton D, et al. Social and psychiatric functioning in adolescents with Asperger syndrome compared with conduct disorder. J Autism Dev Disord 2000;30:279-93.

7. Lugnegård T, Hallerbäck MU, Gillberg C. Psychiatric comorbidity in young adults with a clinical diagnosis of Asperger syndrome. Res Dev Disabil 2011;32:1910-17.

8. Gillott A, Standen P. Anxiety and stress in adults with autism. $J$ Intellect Disabil Res 2004;48:335-35.

9. Gillott A, Standen PJ. Levels of anxiety and sources of stress in adults with autism. J Intellect Disabil 2007;11:359-70.

10. National Institute for Health and Care Excellence. Autism recognition, referral, diagnosis and management of adults on the autism spectrum, NICE Clinical Guideline 142. London: British Psychological Society \& The Royal College of Psychiatrists, 2012.

11. Jarbrink K, Knapp M. The economic impact of autism in Britain. Autism 2001:5:7-22.

12. Jarbrink K, Fombonne E, Knapp M. Measuring the parental, service and cost impacts of children with autistic spectrum disorder: a pilot study. J Autism Dev Disord 2003;33:395-402.

13. Gould RA, Buckminister S, Pollack MH, et al. Cognitive-behavioural and pharmacological treatment for social phobia: a meta-analysis. Clin Psychol Sci Pract 1997;4:291-306.

14. Rowa K, Antony MM. Psychological treatments for social phobia. Can J Psychiatry 2005;50:308-16.

15. Chalfant AM, Rapee R, Carroll L. Treating anxiety disorders in children with high functioning autism spectrum disorders: a controlled trial. J Autism Dev Disord 2007;37:1842-57.

16. Ooi YP, Lam CM, Sung M, et al. Effects of cognitive-behavioural therapy on anxiety for children with high-functioning autistic spectrum disorders. Singapore Med J 2008;49:215-20.

17. Schleismann KD, Gillis JM. The treatment of social phobia in a young boy with Asperger's disorder. Cogn Behav Pract 2011;18:515-29.

18. Sofronoff $\mathrm{K}$, Attwood $\mathrm{T}$, Hinton $\mathrm{S}$. A randomised controlled trial of a CBT intervention for anxiety in children with Asperger syndrome. J Child Psychol Psychiatry 2005;46:1152-60.

19. Wood JJ, Drahota A, Sze K, et al. Cognitive behavioral therapy for anxiety in children with autism spectrum disorders: a randomized, controlled trial. J Child Psychol Psychiatry 2009;50:224-34.

20. Cardaciotto L, Herbert AD. Cognitive behavior therapy for social anxiety disorder in the context of Asperger's syndrome: a single-subject report. Cogn Behav Pract 2004;11:75-81.

21. Weiss JA, Lunsky Y. Group cognitive behavioural therapy for adults with Asperger syndrome and anxiety or mood disorders: a case series. Clin Psychol Psychother 2010;17:438-46. 
22. Spek AA, Van Ham NC, Nykliček I. Mindfulness-based therapy in adults with an autism spectrum disorder: a randomized controlled trial. Res Dev Disabil 2013;34:246-53.

23. Department of Health. Fulfilling and rewarding lives. HM Government, 2010.

24. Roberge $P$, Marchand A, Reinharz D, et al. Cognitive-behavioral treatment for panic disorder with agoraphobia: a randomized, controlled trial and cost-effectiveness analysis. Behav Modif 2008;32:333-51.

25. Power KG, Simpson RJ, Swanson V, et al. A controlled comparison of cognitive behaviour therapy, diazepam, and placebo, alone and in combination, for the treatment of generalised anxiety disorder. $J$ Anxiety Disord 1990;4:267-92.

26. Power KG, Sharp DM, Swanson V, et al. Therapist contact in cognitive behavioural therapy for panic disorder and agoraphobia in primary care. Clin Psychol Psychother 2000;7:37-46.

27. Power KG, Simpson RJ, Swanson V, et al. Controlled comparison of pharmacological and psychological treatment of generalized anxiety disorder in primary care. Br J Gen Pract 1990;40:289-94.

28. Heavens D, Murphy GH, Langdon PE. People with Asperger syndrome and anxiety (PAsSA) trial treatment manual (Version 3.0). Norwich: University of East Anglia, 2012.

29. Connor KM, Davidson JRT, Churchill EL, et al. Psychometric properties of the Social Phobia Inventory (SPIN). Br J Psychiatry 2000;176:379-86.

30. Heimberg RG, Horner KJ, Juster HR, et al. Psychometric properties of the Liebowitz Social Anxiety Scale. Psychol Med 1999;29:199-212.
31. Hamilton $\mathrm{M}$. The assessment of anxiety states by rating. BMJ 1959;32:50-5

32. Rutter M, LeCouteur A, Lord C, et al. Diagnosis and sub-classification of autism: concepts and instrument development. In: Schopler E, Mesibov GB. ed. Diagnosis and assessment in autism, 1988:239-59.

33. Heimberg RG, Mueller GP, Holt CS, et al. Assessment of anxiety in social interaction and being observed by others: the Social Interaction Anxiety Scale and the Social Phobia Scale. Behav Ther 1992;23:53-73

34. Brown EJ, Turovsky J, Heimberg RG, et al. Validation of the Social Interaction Anxiety Scale and the Social Phobia Scale across the anxiety disorders. Psychol Assess 1997;9:21-7.

35. Marks IM, Matthews AM. Brief standard self-rating scale for phobic patients. Behav Res Ther 1979;17:263-7.

36. Hamilton M. A rating scale for depression. J Neurol Neurosurg Psychiatry 1960;23:56-62.

37. Brazier J, Roberts J, Deverill M. The estimation a preference-based measure of health from the SF-36. J Health Econ 2002;21:271-92.

38. Dolan P. Modelling valuations for EuroQol health states. Med Care 1997;35:1095-108.

39. Curtis L. Unit costs of health and social care. Canterbury: University of Kent, Personal Social Services Research Unit, 2009.

40. Department of Health. NHS Reference Costs 2009-2010, 2011.

41. Fenwick E, Claxton K, Sculpher M. Representing uncertainty: the role of cost-effectiveness acceptability curves. Health Econ 2001;10:779-87. 